c4

\section{glutamate}

release

from striatal

cholinergic

neurons has

a previously

unappreciated

role in

mediating

the effects of striatal activity
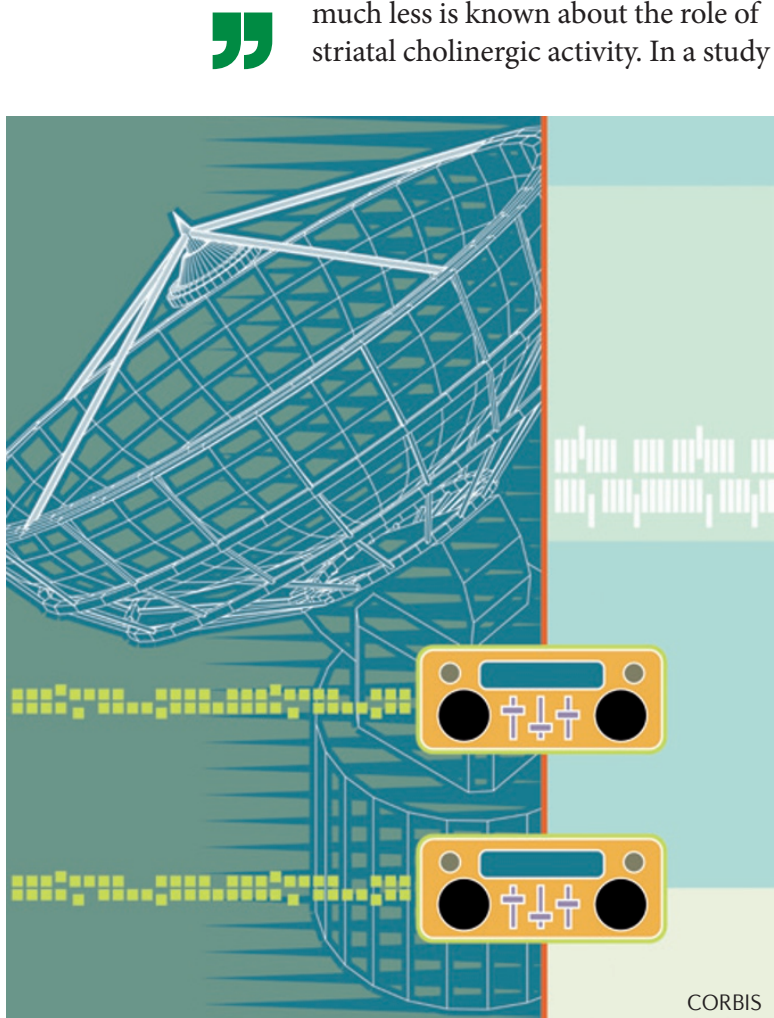

Understanding the regulation of neurotransmission is key to uncovering new strategies for the treatment of motor diseases. Whereas the role of dopamine in the regulation of medium spiny GABAergic neurons (MSNs; the major output neurons of the striatum) is well characterized, much less is known about the role of striatal cholinergic activity. In a study

CORBIS

\title{
Dissecting co-transmission in the striatum
}

published in PLoS Biology, Prado and colleagues dissect the contribution of glutamate release to striatal function by selectively eliminating the vesicular acetylcholine transporter (VAChT) from mouse striatal cholinergic neurons.

Experiments in which cholinergic neurons in the striatum were ablated have demonstrated that they regulate spontaneous motor activity and reward-related behaviours. These cells release two neurotransmitters: acetylcholine and glutamate. Somewhat surprisingly, selective elimination of VAChT, and thus cholinergic transmission, in the mouse striatum had minimal behavioural consequences. Mutant mice showed no deficits in motor performance or motor learning. Moreover, their spontaneous locomotor activity did not differ from that of wild-type mice. Previous experiments implicated striatal cholinergic neurons in the rewarding effects of cocaine, but VAChT-deficient mice did not display cocaine-induced hyperactivity or its rewarding effects, as measured by a conditioned-place preference paradigm. These results indicate that glutamate release from striatal cholinergic neurons has a previously unappreciated role in mediating the effects of striatal activity.
Interestingly, in VAChT-deficient mice, MSNs showed increased sensitivity to dopamine receptor agonists. This is likely to be due to the increased expression of dopamine receptors in the striatum, as no changes in the levels of dopamine or its metabolites were detected. This finding could have important implications for the treatment of conditions such as Parkinson's disease, which is characterized by deficient dopaminergic function and high striatal cholinergic activity.

By selectively eliminating VAChT in the striatum without disrupting glutamate neurotransmission, the authors were able to highlight the contribution of glutamate to striatalrelated behaviours and, more generally, to show how a single population of neurons can use distinct neurotransmitters to differentially regulate a particular circuit. Finally, the enhanced dopaminergic signalling that results from targeting VAChT in the striatum opens up a new avenue in the search for more effective treatments for Parkinson's disease. Monica Hoyos Flight

ORIGINAL RESEARCH PAPER Guzman, M. S. et al. Elimination of the vesicular acetylcholine transporter in the striatum reveals regulation of behaviour by cholinergic-glutamatergic co-transmission. PLoS Biol. 9, e1001194 (2011) 